\title{
Bidirectional Communication System on Power Line Integrated on Electronic Board for Driving of LED and HID Lamps
}

\author{
P. Visconti, ${ }^{1}$ S. D'Amico, ${ }^{1}$ A. Baschirotto, ${ }^{2}$ D. Romanello, ${ }^{3}$ P. Costantini, ${ }^{1}$ \\ V. Ventura, ${ }^{1}$ and G. Cavalera ${ }^{3}$ \\ ${ }^{1}$ Department of Innovation Engineering, University of Salento, 73100 Lecce, Italy \\ ${ }^{2}$ Department of Physics, University Bicocca, 20126 Milano, Italy \\ ${ }^{3}$ Electronic Division, Cavalera Sistemi s.r.l., Galatone, 73044 Lecce, Italy
}

Correspondence should be addressed to P. Visconti, paolo.visconti@unisalento.it

Received 30 June 2011; Revised 13 October 2011; Accepted 18 October 2011

Academic Editor: C. M. Liaw

Copyright (c) 2012 P. Visconti et al. This is an open access article distributed under the Creative Commons Attribution License, which permits unrestricted use, distribution, and reproduction in any medium, provided the original work is properly cited.

\begin{abstract}
We present the bidirectional power line communication system developed in parallel to an electronic board for driving and control of HID (high-intensity discharge) and LED (light-emitting diode) lamps. The communication system, developed to be applied in the sector of public illumination, is been designed to combine high efficiency and reliability with low production costs; it consists indeed of discrete cheap components. The communication system described in this paper implements the technique of transporting digital information over existing power lines, avoiding the issue of installing new cables. Digitized signals can use power line cables through the amplitude voltage and current modulation. The solution proposed is more advantageous compared to communication techniques currently on the market which are essentially two types, power line carrier (modem for high-voltage lines) or radio (zig-Bee transceiver).
\end{abstract}

\section{Introduction}

Public lighting represents a primary service in the management of a city. It is indeed public safety guarantor, helps to improve the environment in which people live, and promotes city image through the enhancement of cultural heritage. Geographical extension of public lighting system makes it very difficult in order to ensure proper levels of efficiency, quality, reliability, and energy savings of the service by using traditional instruments.

An intelligent electronic system for the control of both LED and HPS lamps for public lighting was designed. The feature of driving both types of lamps is unique for the realized system. This versatility is achieved through the implementation of a reconfigurable output capable of driving, by the user's choice, a LED or HPS lamp. Through an appropriate routine implemented in the firmware of the microcontroller, the system is capable of self-learning the type of lamp inserted in reconfigurable output. This feature allows to use the ballast in lighting systems that nowadays use the traditional discharge lamps and, primarily, will allow to keep the same ballast when discharge lamps will be replaced by the LED modules, in the near future in which the LED street lighting systems will be more affordable.

The lighting implants are indeed characterized by a large number of hot spots scattered throughout the territory for which it is constantly necessary to monitor the operation and make its management. Particularly in urban areas, public lighting systems are greatly extended and widely distributed, and they contain a large number of control panels and, above all, light points. Therefore, it is extremely problematic and expensive to make early detection of malfunctioning components, to locate and remove the cause of failures, to carefully manage the lighting implant, and to control the luminous flux of each unit. All this involves high costs in terms of time and money.

The remote control is a system designed for managing lighting systems and helps to combine cost savings with safety and service continuity. The main advantage is the 


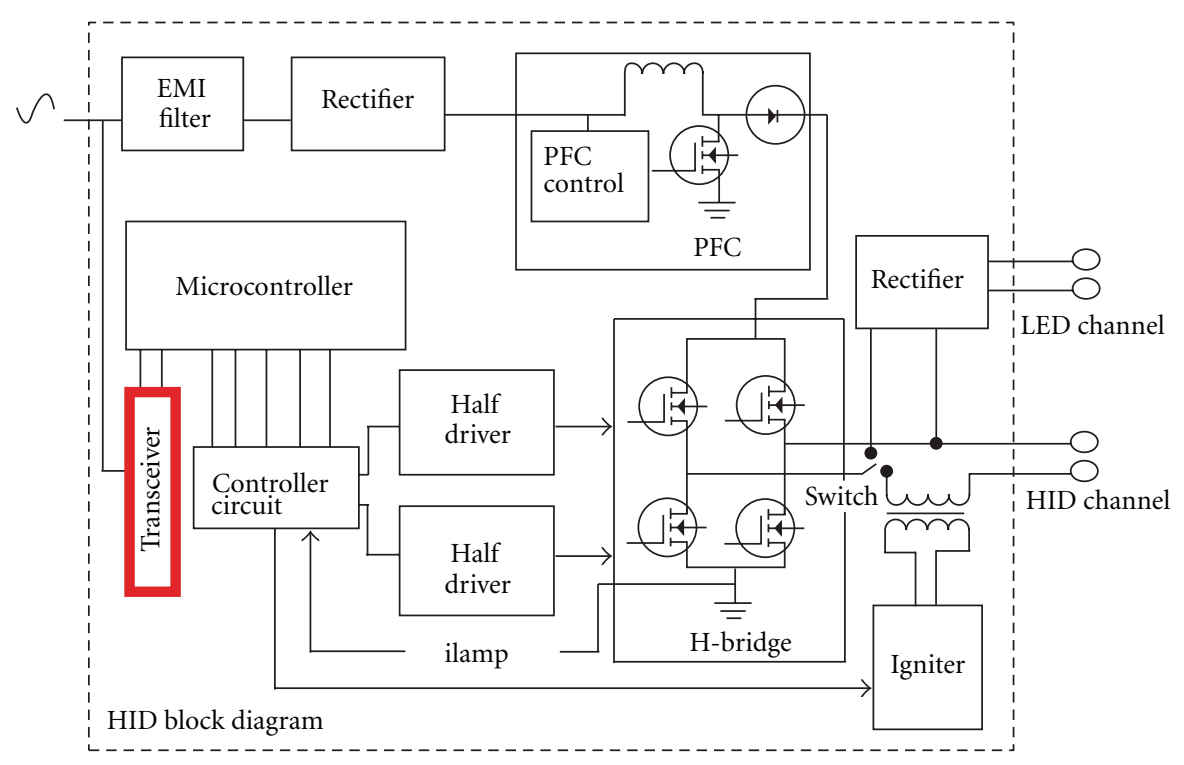

FIGURE 1: Electronic board block diagram.

implant's control in real time plus conditions improving of maintenance (i.e., real-time report of malfunctions). The remote control allows

(i) eliminating unnecessary costs due to the troubleshooting,

(ii) transparency, reduced operating costs, and warehouse management,

(iii) automatic service regulation in the seasonal demand,

(iv) real-time report on plants disruption and therefore the possibility of quick intervention to restore normal conditions,

(v) scheduling of major repairs,

(vi) human resources and emergency response teams optimization.

The designed transceiver system was thought to allow bidirectional communication from the control panel (master) to the individual light points (slave), using the power line as transmission medium in order to get remote control and regulation of the light intensity produced by each lighting point, as required by actual regulations regarding light pollution and energy saving.

The designed transmission system, applied to a public lighting system, allows transmission of information between the control panel and the lighting points, through the power line; consequently it is not required the installation of additional cables. This peculiarity, together with the use of relatively economical discrete components for the realization of the transmitter and receiver units, ensures low production costs, simple construction and maintenance of the system and a good overall reliability; this is certainly the main advantage of the designed communication module.

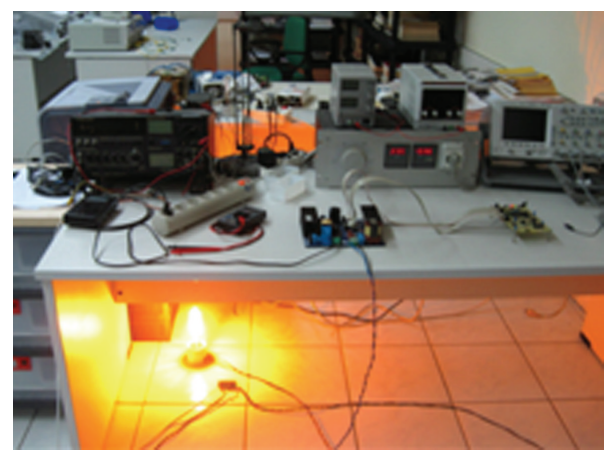

FIGURE 2: Experimental test.

\section{Electronic Board Designed for Driving HID and LED Lamps}

This section provides a brief description of the designed electronic ballast. Figure 1 shows the complete block diagram of the electronic ballast for HID and LED lamps, with the communication module highlighted. This electronic system is designed to power-supply a discharge lamp or LED lamp. The developed communication system implements the master-slave mechanism; therefore, the transceiver inserted into the ballast is the slave module. The power factor corrector (PFC) optimizes power consumption by the power line, avoiding unnecessary loss of power and provides a stabilized high voltage (Vbus). The output stage, consisting of an H-bridge, is driven by two half-bridge drivers and allows the lamp to be powered by an $\mathrm{AC}$ and low-frequency signal. The drivers are driven by a controller circuit which has the task of controlling the current in the lamp.

The microcontroller provides the PWM signals to the controller circuit. The microcontroller, installed on the ballast, is intended only to generate square-wave signals 


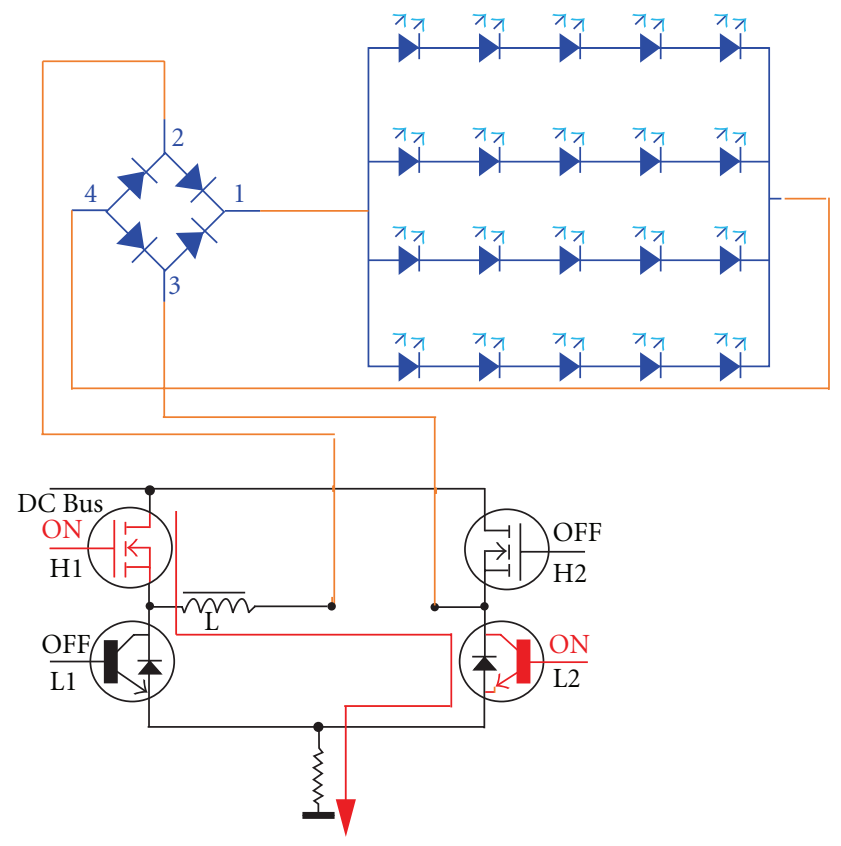

FIGURE 3: Led module driver circuit.

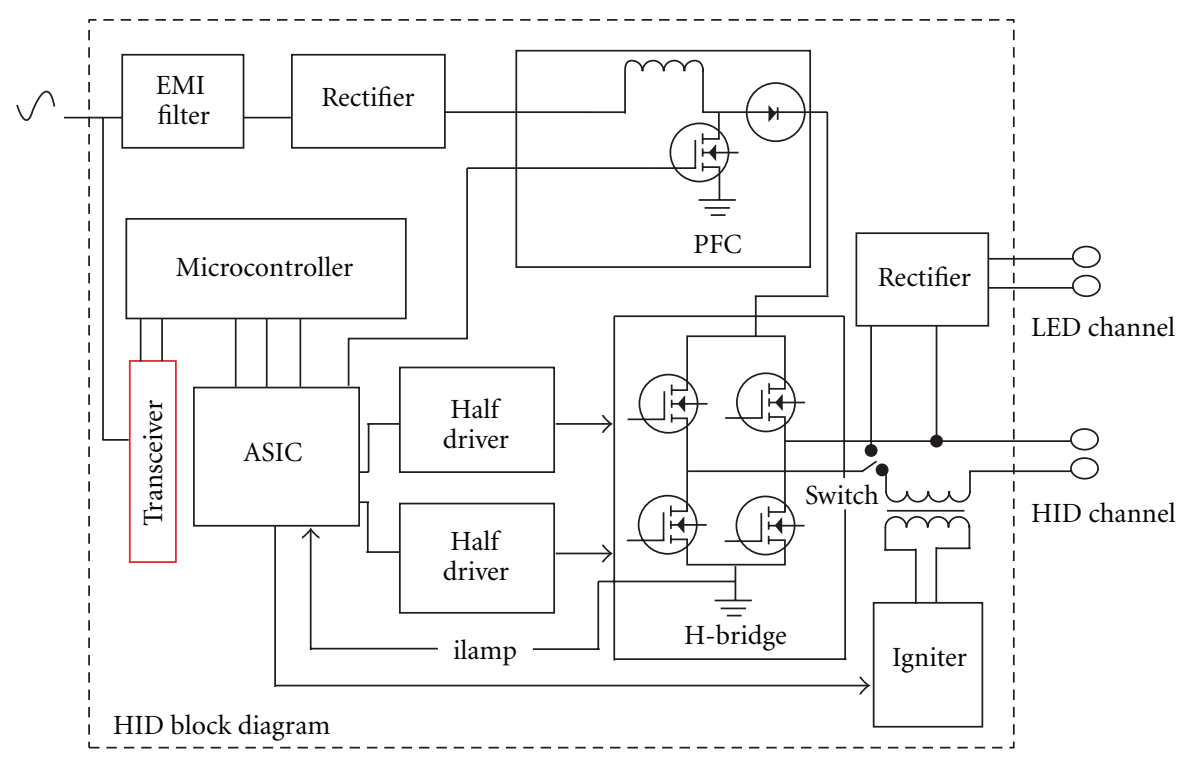

FIgURE 4: Electronic board block diagram with ASIC solution.

to drive the H-bridge and the PWM signal in order to adjust the current in the lamp, according to the commands received from the electrical cabinet through the transceiver module. The heart of the ballast is the controller circuit: it is composed of operational amplifiers, comparators, and a programmable device (GAL). The GAL implements a logic circuit which has the aim of adjusting the current flowing in the HID/LED lamp, on the basis of signals provided by the microcontroller and the feedback from the powered lamp. The fact that this feedback does not pass through the microcontroller guarantees a certain robustness of the system. The attractive aspect of the designed board is the possibility to drive both HID and LED lamps with the same driver output. This is possible using a switch to remove temporarily the igniter circuit for LED lamp. On the LED channel is present a rectifier bridge in order to rectify the AC voltage from the H-bridge. Another important feature is the phase of autolearning of the type of load placed on the reconfigurable output of the ballast. Supplying the load with a voltage higher than the $V_{\text {forward }}$ of the eventual inserted LED module, the system begins to read the current flowing on the same load; if a current value is detected, an LED lamp 


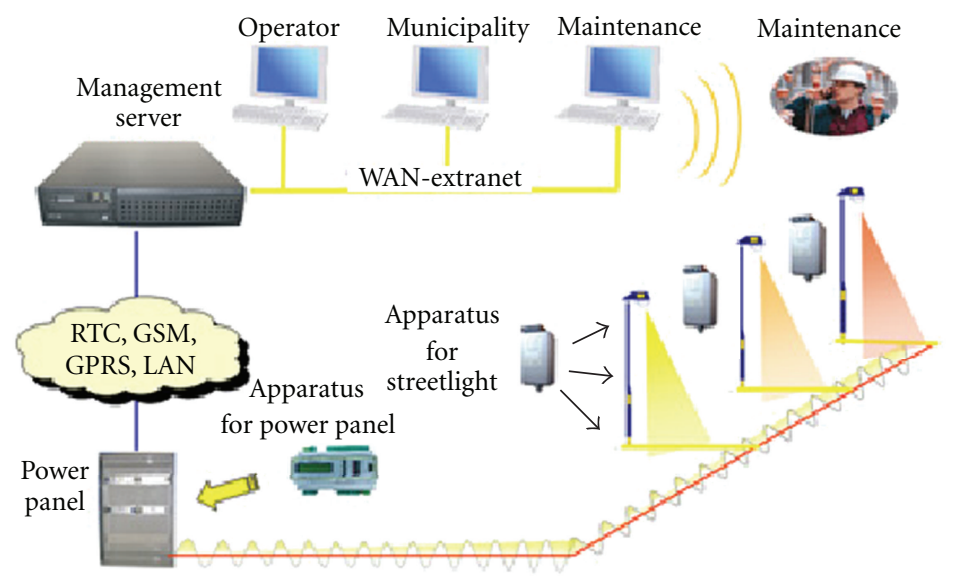

FIgURE 5: Light point to light point control module.

is connected, and if no current is detected, it means that an HID lamp has been inserted or the channel is empty (i.e., when the HID lamp is not triggered it is an open circuit). At this point, if the load is an LED module, the process performs the soft start phase, in which a ramp current on the LED load is provided until the rated current of the LED module is reached. Once this phase is over, the load current is kept constant (burn phase). If, instead, the inserted load is recognized as an HID lamp, the first phase to perform is to create the ignition pulse of $4-5 \mathrm{kV}$ across the lamp. If the lamp ignites, a warm-up phase is performed with the purpose to keep the current constant, while the voltage reaches the lamp nominal value. After this stage, the power dissipated by the lamp is kept constant (burn phase).

The designed electronic system has been tested by triggering and supplying a $150 \mathrm{~W}$ discharge lamp (Figure 2). By means of a DIP switch connected to the microcontroller it was possible to change the current in the lamp. In according to the state of the DIP switch, in fact, the microcontroller varies the duty cycle of the PWM signal which has the task of regulating the current in the lamp. As expected, a variation of the luminous flux produced by the lamp was observed by changing the status of the switches.

For driving with the same driver of both HID and LED lamps, the principle idea is using the $\mathrm{H}$-bridge for supplying LED lamps. This type of lamps is completely different from HID lamps, and they do not require ignition circuit (disabled for this application). In Figure 3, we show an example of a LED module driven by modified $\mathrm{H}$-bridge through diodebridge rectifier.

The designed electronic ballast, in case of power supplying of LED module, is not very efficient. Its main application is in fact relative to the power supply of HID lamps; in this case the system's efficiency is above $90 \%$, as discussed elsewhere [1-3].

We have also designed an application-specific integrated circuit (ASIC), which integrates some devices installed on the ballast for HID and LED lamps, for minimizing the cost of the board and PCB area. It is fabricated using AMS CMOS $0.35-\mu \mathrm{m}$ technology. Then it was designed another driving

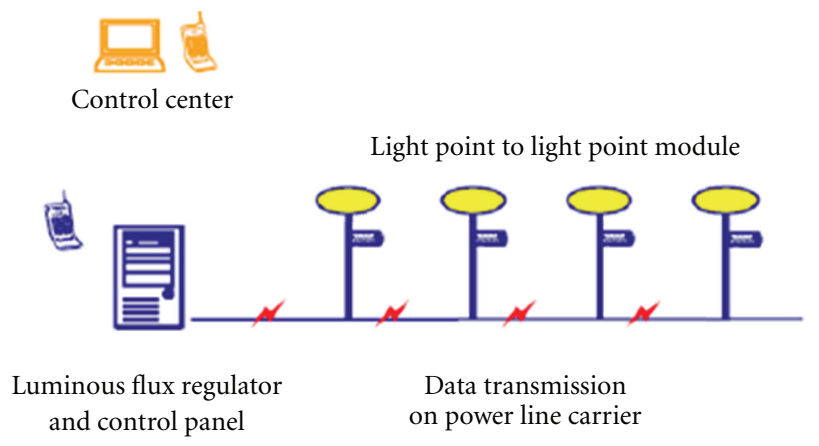

FIgURE 6: Light point to light point functional diagram.

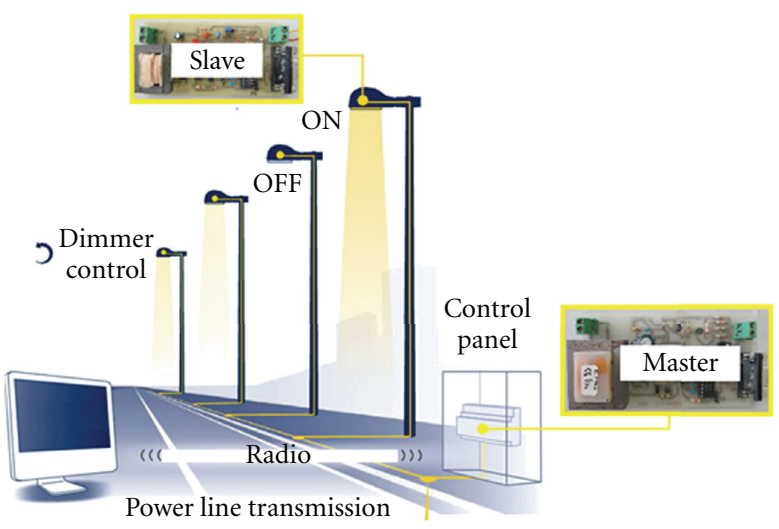

FIGURE 7: The communication system that we have designed, applied to a lighting system: the master will be placed in control panel, and the slave will be installed in the individual light points.

board with the fully integrated ASIC that incorporates the controller circuit to adjust the current in the lamp and the control circuit of the PFC (Figure 4).

The microcontroller, through the communication module, receives the command from the electrical cabinet for adjustment of luminous flux of the lamp. Communication between the microcontroller and the ASIC is through the 


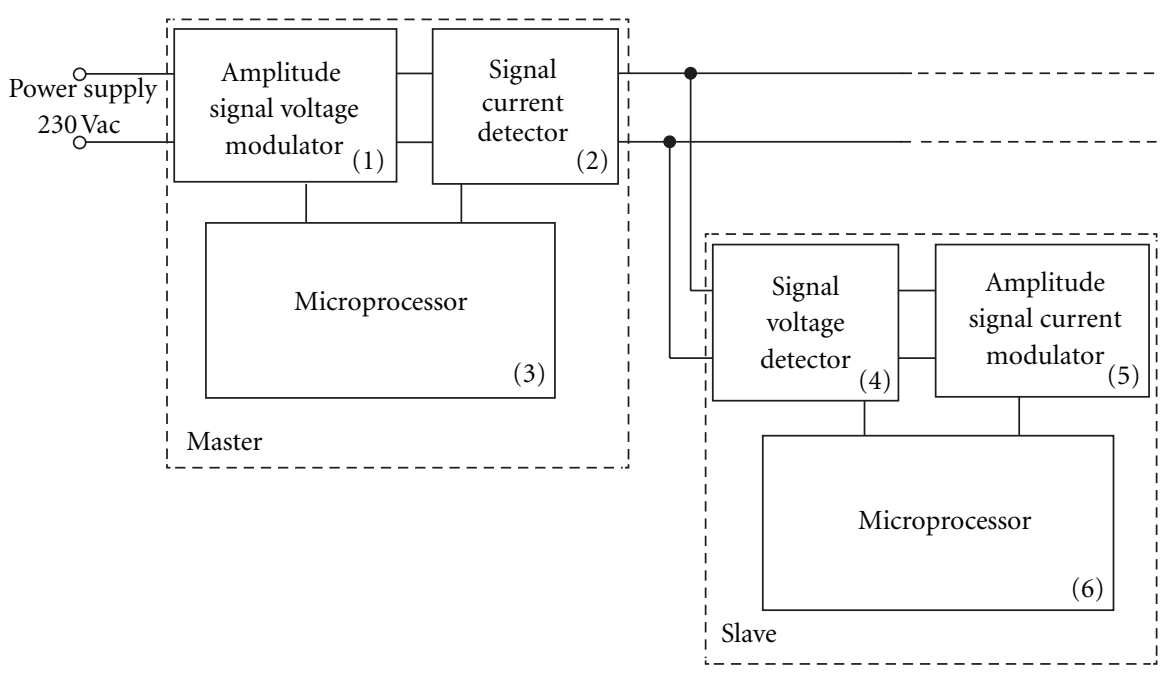

Figure 8: Transceiver block diagram.

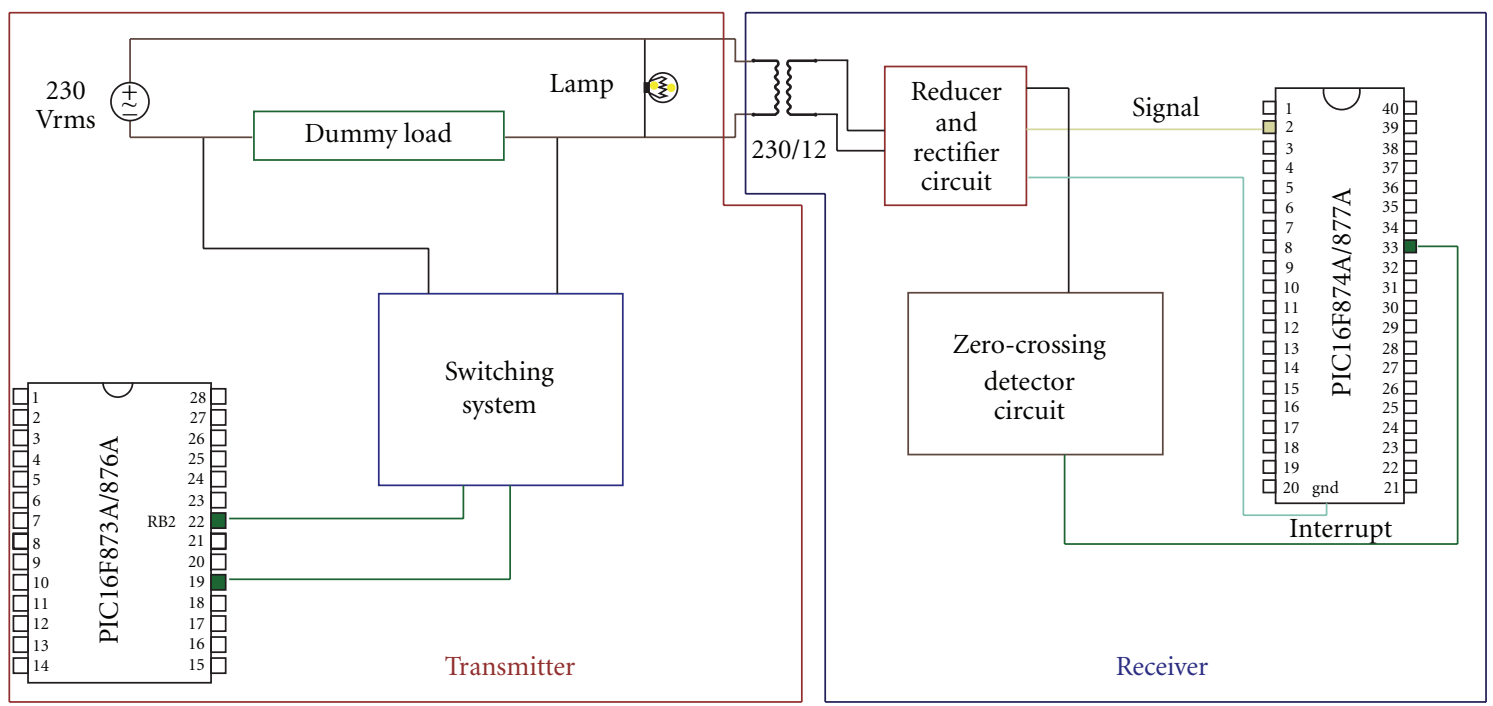

Figure 9: Simplified schematic of the master-to-slave communication system.

SPI serial communication protocol. The microcontroller provides to the ASIC the appropriate signals for the current adjustment in the lamp.

\section{3. "Point-to-Point" Light Control System}

This remote control system consists of electronic equipments for monitoring, management, and control of individual lamps; it is based on power line carrier technology that enables digital communication between the module installed on a single light point (located in the cockpit, in the terminal, or in the luminaire itself) and the managing server, placed within the command framework (Figure 5).

In actual power line communication system, the digital data are modulated (frequency modulation) on power supply; so there is no need of additional pipes/cables in the implant. By means of this system, it is possible monitoring and control of the electrical parameters of the individual lamps, identifying faults and alarms, turning on, turning off, minimizing the consumption, or adjusting the intensity of individual lamps using manual or automatic controls. Single light-point electrical information is transmitted periodically and stored in the control panel. By using the management software, the control system acquires the measurements and generates events and alarms based on customizable criteria. It's possible therefore managing from a central unit the single lighting point, providing an intelligent control of lighting (Figure 6).

\section{Communication System: Design Concept}

In this section, we present the work on the communication system between the control panel and the electronic ballast for HID and LED lamps. Designed solution is a modification 


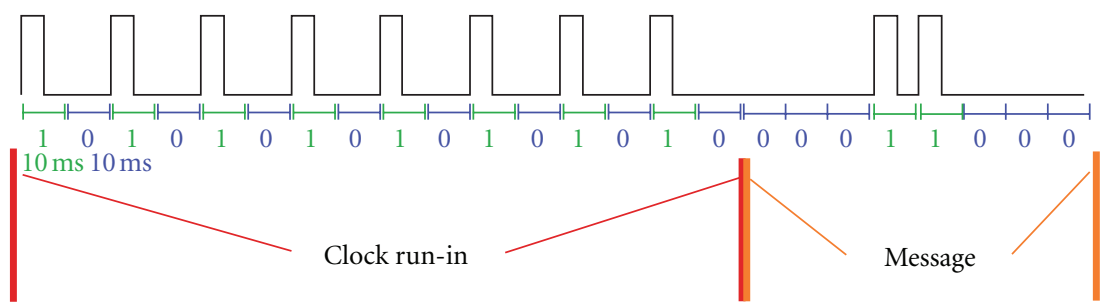

Figure 10: Signal sent from the transmitter to the receiver (clock run-in + message).

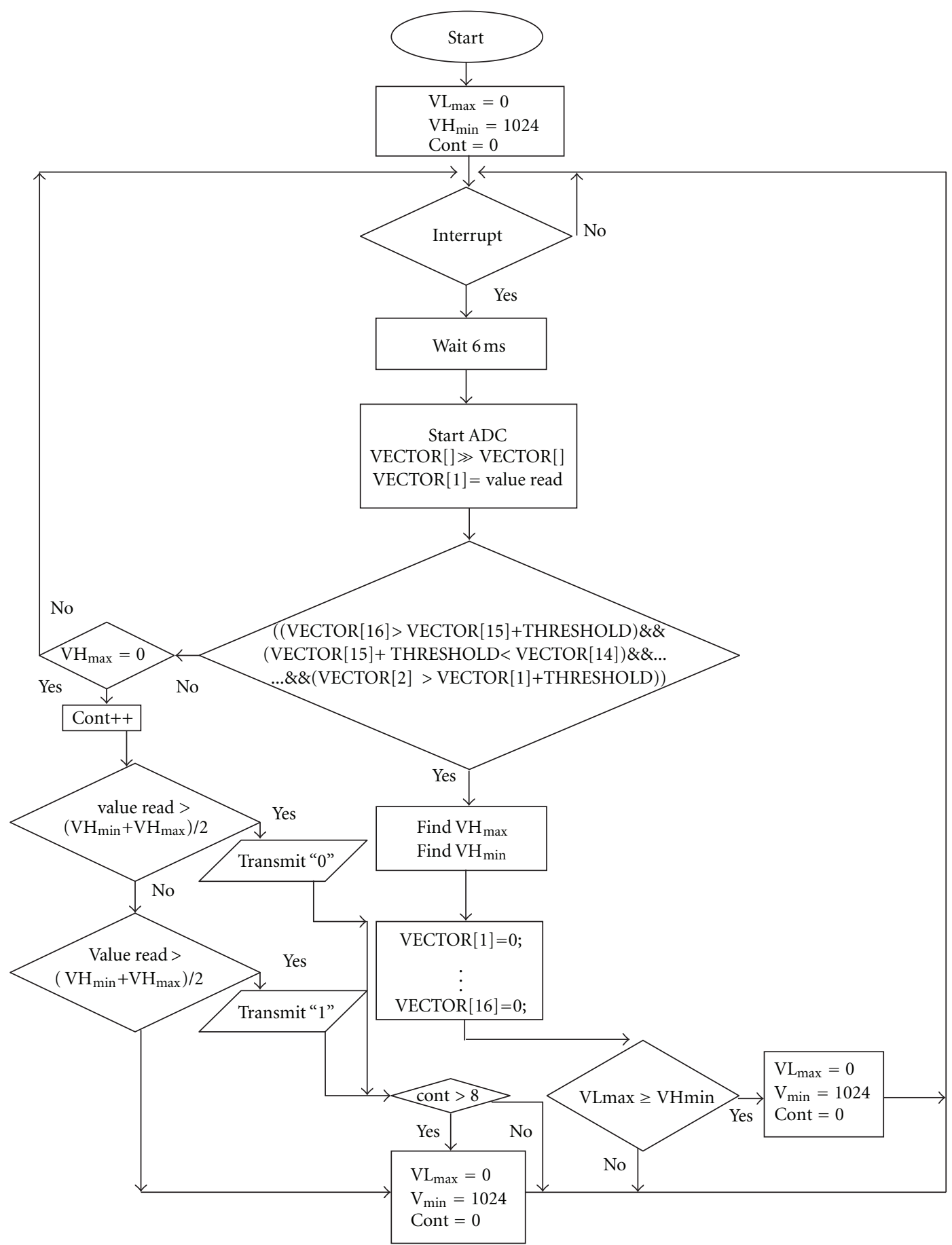

FIGURE 11: Algorithm of the receiver firmware. 
of the M-Bus system characterized by the transmission between master and slave on two wires, so that communication between control panel (master) and the electronic ballast of each lamp (slave) can be realized on the power line (Figure 7). As explained above, the simplicity and low cost of this solution, in addition to good efficiency and reliability, makes it innovative compared to communication techniques currently on the market.

4.1. Block Diagram of the Communication System. The block diagram of the communication system is shown in Figure 8. The communication from master to slave is achieved by amplitude modulation of the voltage signal (by means of amplitude signal voltage modulator block); the communication from slave to master is obtained by modulating the current signal with amplitude signal current modulator block.

\section{Master-to-Slave Communication}

The master-to-slave communication is realized through the voltage modulation on the power line.

\subsection{Master-to-Slave Communication: Block Diagram. The} transmission of logical 0 or 1 is generated by a microcontroller installed on the master, properly programmed to produce a digital signal that can control a switching system, that handles the insertion of a dummy load on power line, in series with the lamp. The connection of the dummy load on the line causes a voltage drop and consequently a lower network voltage on the load lamp. The network signal consists of a sine wave voltage with $230 \mathrm{Vrms}$ and $50 \mathrm{~Hz}$; so the transmitter PIC's digital signal is synchronized with the wave period, in order to modulate the peak values. In this way, when the dummy load is connected, we obtain reduced peak values, corresponding to transmission of a logical zero; instead, when the dummy load is not connected, we obtain unchanged peak values, corresponding to transmission of a logical one. The reception is performed by a second PIC installed on the slave, after reduction and rectification of the signal from the power line (Figure 9). The receiving PIC, synchronized by an appropriate interrupt signal, is programmed to receive this signal, to acquire the peak values and to convert them into digital format for software processing. The intelligent device, using an algorithm dedicated to this function, is thus able to discriminate the transmitted logic level.

The slave-to-master communication is achieved by modulating the current on the power line, by arming and disarming of an additional load in parallel with the lamp of each light point. When the load is engaged, the power line current is greater than when the same load is not connected. On each light source is installed a microcontroller that, suitably programmed, manages the placement of the load according to the bits to be transmitted. In order to transmit a logical one, it inserts the load by activating the switch, through the dedicated output pins; instead to transmit a logical zero, the output pin does not allow the activation of the switch.

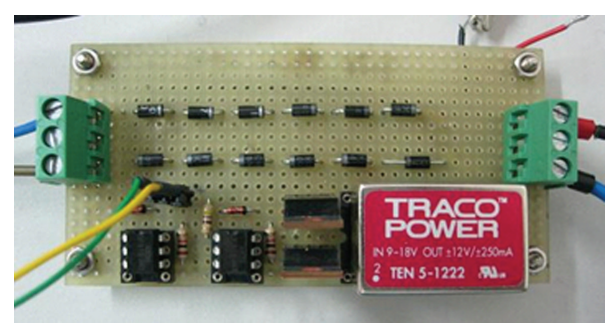

FIGURe 12: Modulator circuit voltage.

5.2. Master-to-Slave Communication: Algorithm Operation. Each data packet transmitted from the modulator begins with a sequence of bits called clock run-in (CRI). This sequence is designed to "announce" the start of the message and to inform the demodulator about the actual voltage level corresponding to the logical zero and logical one (depending on variation of $50 \mathrm{~Hz}$ network power voltage caused by different loads). The sequence of bits of the CRI in transmission is 1010101010101010. In order to carry out the experimental tests, in the packet to send, immediately after the CRI sequence, it was added, for example, the message 00011000 (Figure 10). The receiver will recognize the message transmitted according to the voltage levels provided by the CRI. The microcontroller installed in the modulator, therefore, has been programmed with a firmware capable of reproducing the signal of Figure 11.

The algorithm implemented in the firmware of the receiver is shown in Figure 11.

The receiver captures the peak voltage every $10 \mathrm{~ms}$. The acquired samples are inserted into a vector (VECTOR []) in order to recognize the clock run-in.

The receiver, once recognized the clock run-in, must subsequently interpret the logic levels, 0 and 1 , that characterize the message transmitted. Between the elements of vector containing the CRI are detected $\mathrm{VH}_{\text {min }}$ (minimum level of quantization in order to recognize the sample captured as logical 1) and $\mathrm{VL}_{\max }$ (maximum level of quantization in order to recognize the sample captured as a logical 0 ). We defined a discriminating cutoff between the set of samples corresponding to the logical zero and those corresponding to a logical one, the half sum between $\mathrm{VL}_{\max }$ and $\mathrm{VH}_{\min }$. Essentially, if the value acquired after the CRI is greater than or equal to $\left(\mathrm{VL}_{\max }+\mathrm{VH}_{\text {min }}\right) / 2$, it is decoded as a logical one; if it is lower, consequently it is decoded as a logical zero.

5.3. Master-to-Slave Communication: Experimental Test. We have realized on breadboard the transmitter circuit consisting of the dummy load and the switching system. In Figure 12 is shown an image of the obtained electronic board.

In Figure 13 is illustrated the experimental set up of the communication test from master to slave.

In the yellow box, there are the components of the transmitter side, in particular the modulator circuit just described. The electronic board below in the yellow box, realized by Cavalera Sistemi for other applications, has been used only to the benefit of the PIC installed on it. The 


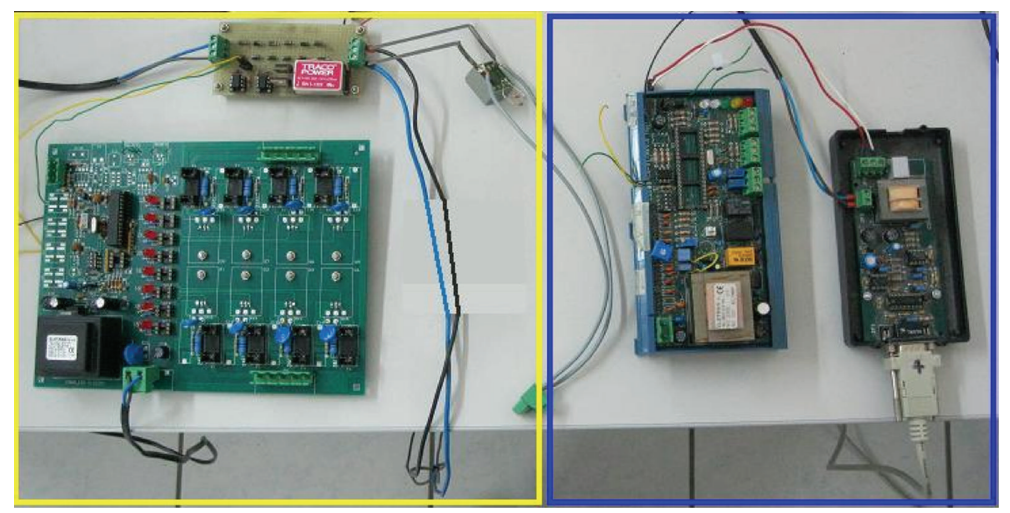

FIGURE 13: The workbench with the designed communication system.

output voltage from the modulator (master) is sent to an incandescent lamp that represents the load on the power line. In the blue box, there is the circuitry concerning the receiver (slave). In addition to the CS083 electronic board, which contains the PIC and the zero-crossing detector circuit, there is also a RS485-RS232 converter for serial communication with the PC.

At this point, we tested the master-to-slave communication system by sending different massages and checking the received ones to the slave receiver. It was revealed that $100 \%$ of the transmitted messages has been correctly decoded (sent message 00011000 after each clock run-in). Afterwards, it was decided to verify the behavior of the communication system dynamically changing the message to be transmitted. For this scope, it was modified the firmware in the transmitter in order to send a cyclical count from 0 to 255 . The test result was positive $(100 \%$ of the sent messages correctly received).

Subsequently, we proceeded to test the robustness of the designed system with respect to external disturbances, such as variations of network power voltage in the range of $20 \%$ in negative and positive, simulated in experimental test by the action of the variac control, or the presence of a source of noise, generated in the test with an electric trepan connected to the same communication line. In Figure 14 is reported the shapes of transmitted digital signal and of the modulated signal. The difference between the peak values of half waves with modulation (logical 0) and without modulation (logical 1) is about 35$40 \mathrm{mV}$, over 20 quantization intervals for internal PIC 10 bit ADC.

\section{Slave-to-Master Communication}

The slave-to-master communication is obtained by modulating the current on the power line, by arming and disarming of an additional load in parallel with the lamp (Figure 15).

\subsection{Slave-to-Master Communication: Block Diagram. When} the load is engaged, the power line current is greater than when the same load is not connected. The produced current variation is read by the control station through

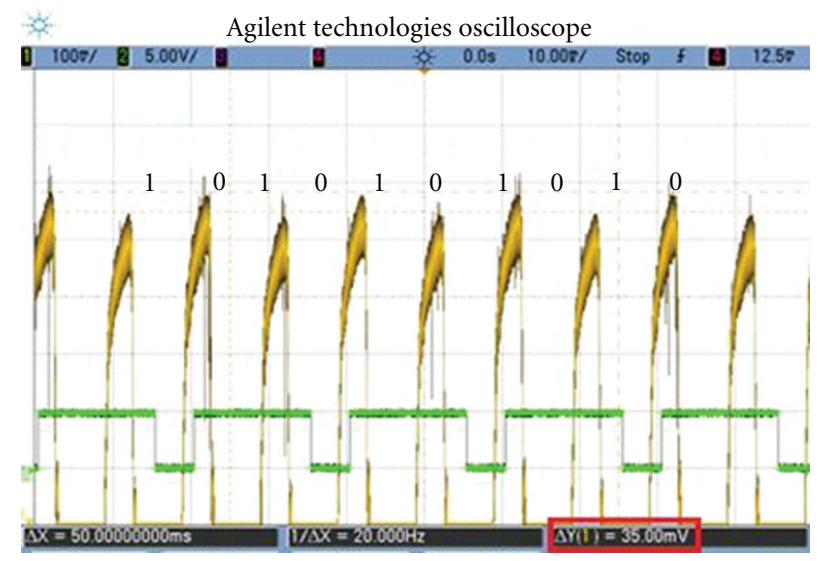

FIGURE 14: The signal generated by the transmitter (green) and the modulated voltage signal (yellow).

a bidirectional current sensor, which produces an output voltage signal proportional to the measured current. The input-output characteristic of the current sensor is as follows. For zero current, the output is equal to $2.5 \mathrm{~V}$. For positive currents the output changes to $(2.5 \mathrm{~V}+\Delta \mathrm{V})$. Instead for negative currents, the output changes to $(2.5 \mathrm{~V}-\Delta \mathrm{V})$. Therefore, the sinusoidal input current signal produces a sinusoidal output voltage signal, centered on the value of $2.5 \mathrm{~V}$. This signal is then processed by the receiver microcontroller, that, thanks to a proper timing by the interrupt signal, captures the peak values and converts them into digital format for software processing. The PIC, properly programmed, is able to discriminate the transmitted logic level. In the slaveto-master transmission, the object of modulation, that is, the current, varies depending on the number of powered lamps; if this number increases, the current that has to be modulated increases too. In order to manage this dynamic situation, maintaining a good resolution in the transmission, it is necessary only to change the reference voltages of the $\mathrm{AD}$-Converter of the microcontroller for different lighting plants.

6.2. Slave-to-Master Communication: Algorithm Operation. Regarding the algorithm of the transmitter in order to carry 


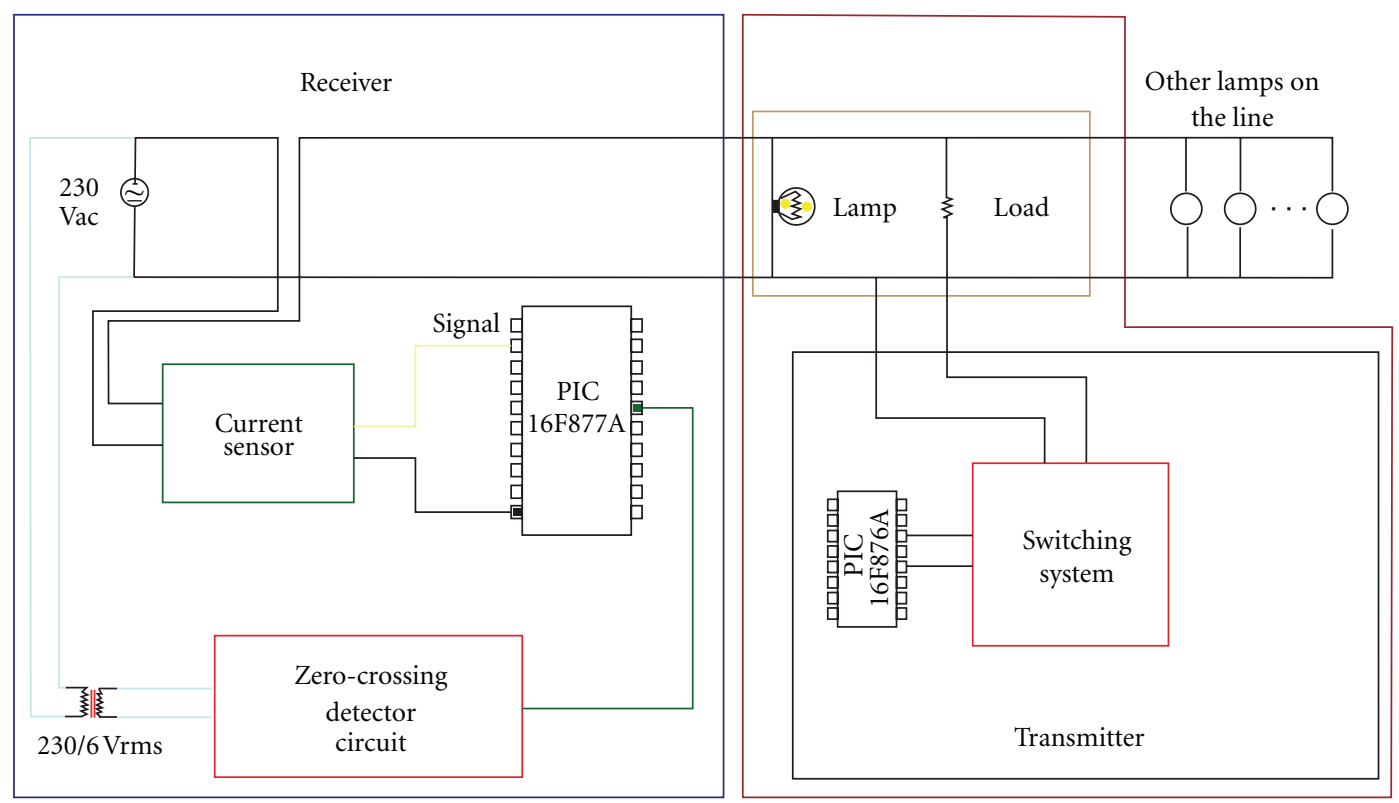

FIGURE 15: Simplified schematic of the slave-to-master communication system.

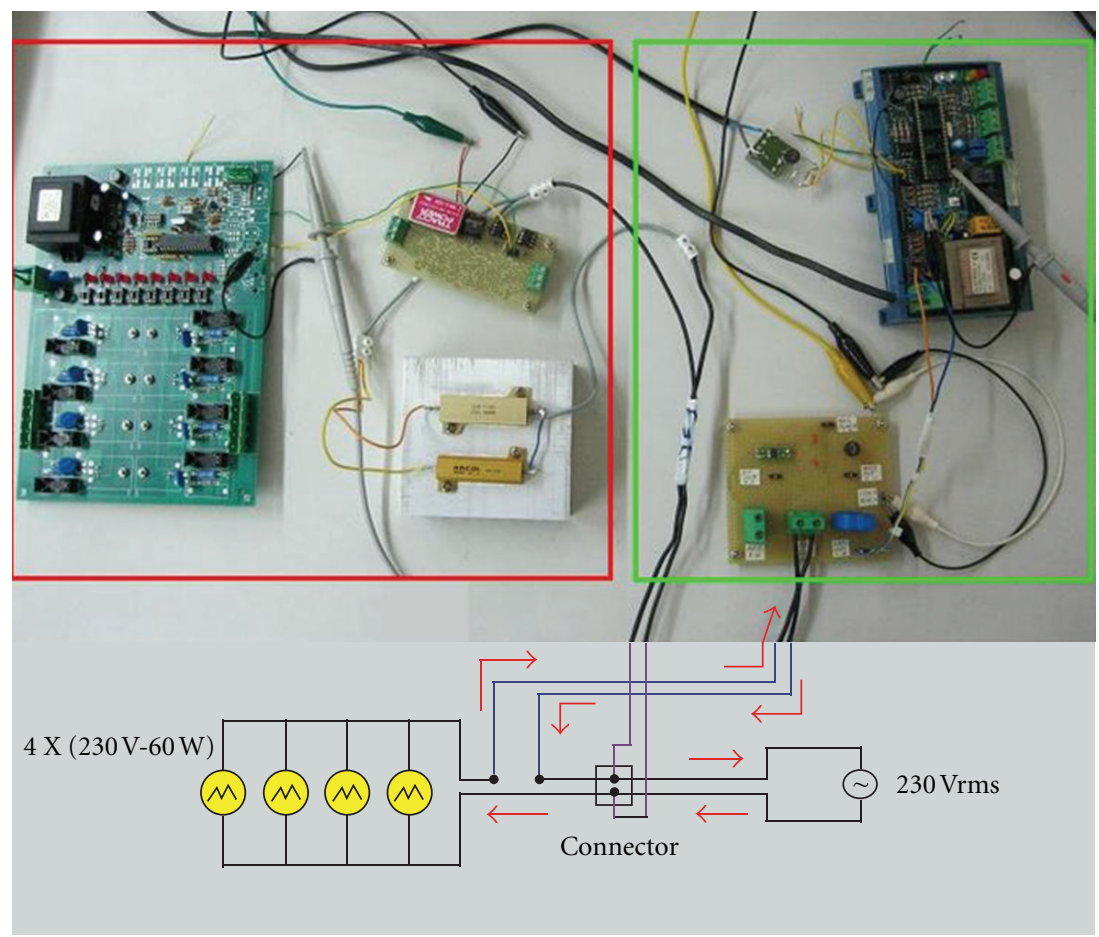

FIGURE 16: The "slave-to-master" communication system.

out the transmission tests in the laboratory, the PIC is programmed in order to generate the message shown in Figure 11. Due to the nature of the voltage signal provided by the current sensor, it was appropriate in this case to modulate the signal during the whole period, that is, every $20 \mathrm{~ms}$. This of course implies a lower transmission rate. Regarding the receiver firmware, however, the only variation from to the algorithm of Figure 12 is due to an additional control; this modification was necessary because the PIC does not recognize all the peaks of the half waves but only the superior peaks.

6.3. Slave-to-Master Communication: Experimental Test. An experimental test was carried out for the communication from slave-to-master. In order to get $1 \mathrm{~A}$ of current in the communication line (as equivalent to one lighting point 
turned on in a public lighting system), we used a load consisting of four $230 \mathrm{~V} / 60 \mathrm{~W}$ lamps connected in parallel. The slave-to-master communication system on the test bench is shown in Figure 16.

The transmitter side (contained in the lighting point) was evidenced in the figure by a red border, while the receiver side (contained in the control cabinet) with a green border. The red border shows the solution used for the resistive load that is connected/disconnected to modulate the current; it consists of two resistors in parallel, which realize an equivalent resistance of $500 \mathrm{Ohm}-100 \mathrm{~W}$. The resistors, fixed on a heat sink, are connected to the power line through the switching circuit. When they are connected to the power line, the additional resistive load will cause a current increase of $0.5 \mathrm{Amp}$ (from $1 \mathrm{Amp}$ to $1.5 \mathrm{Amp}$ ), and the sensor current will detect this variation.

The transmitter has been programmed to send the binary digits of 8 bits, for a cycle count from 0 to 255 . It was observed that the transmission was successful $(100 \%$ of the sent messages correctly received). The test was repeated simulating the large swings of the voltage on power line; even in these cases we have obtained satisfactory results.

\section{Conclusion}

We have designed and developed a communication system, on the power line, capable of allowing the bidirectional communication between the control panel (master) of a lighting plant and the single lighting point (slave). Slaveto-master communication is implemented modulating the voltage amplitude, while the slave-to-master communication is done modulating the current amplitude. This communication system has been tested in the laboratory, in both directions, connecting through cable lines long about two meters, slave circuits with the master electronic board. We have carried out several tests with different loads on the power line, in order to simulate the presence of one or more light sources (slave). Regarding the master-toslave communication, being the lighting points connected in parallel and the communication carried out by means of amplitude modulation of the voltage, there are no limitations for the number of slaves that the system can support.

Instead, in the slave-to-master transmission, since the modulation occurs on the current, we must consider the number of lighting points on the line. In fact, according to the number of light points on the line, different solutions have been studied in order to keep the system reliable; at the moment, we achieved the control up to 40 lighting points. The baud rate in Master to Slave transmission is 100 baud; instead, the baud rate in slave-to-master transmission is 50 baud. The designed system was tested in the laboratory obtaining satisfactory results, even in the presence of noise $[4,5]$. The next step is the test of the communication system on a real lighting system to determine the technical limits (transmission distance, number of slave modules supported, baud rate error, etc.) of the designed communication system.

\section{Acknowledgments}

Intervention was cofinanced by the E.U.-E.R.D.F. on the O.P. Apulia Region 2007-2013-Axis I-Line 1.1 "Aid for investment in research for SMEs" and supported by Cavalera Sistemi Srl-Galatone (LE).

\section{References}

[1] P. Visconti, D. Romanello, G. Zizzari, and G. Cavalera, "Electronic board for driving of HID and LED lamps with auxiliary power supply from solar panel and presence detector," in Proceedings of the 10th International Conference on Environment and Electrical Engineering (EEEIC.EU'11), pp. 430-433, Rome, Italy, May 2011.

[2] P. Visconti, D. Romanello, G. Zizzari, and G. Cavalera, "Multichannel electronic board for control of LED and high intensity discharge lamps," in Proceedings of the International Conference on Industrial Electronics and Systems Engineering (ICIESE '11), Venice, Italy, April 2011.

[3] A. Costantini, D. Romanello, P. Visconti et al., "Electronic board for driving and control of HID and LED lamps in lighting systems," in Proceedings of the 4th International Conference on Sensing Technology (ICST '10), S. C. Mukhopadhyay, A. Fuchs, G. S. Gupta, and A. Lay-Ekuakille, Eds., pp. 199-204, University of Salento, Lecce, Italy, June 2010.

[4] X. Carcelle, Power Line Communications in Practice, Electricité de France, 2009.

[5] H. Ferreira, H. Grove, O. Hooijen, and A. J. H. Vinck, "Power line communication," in Encyclopedia of Electrical and Electronics Engineering, J. G. Webster, Ed., pp. 706-716, WileyInterscience, New York, NY, USA, 1999. 

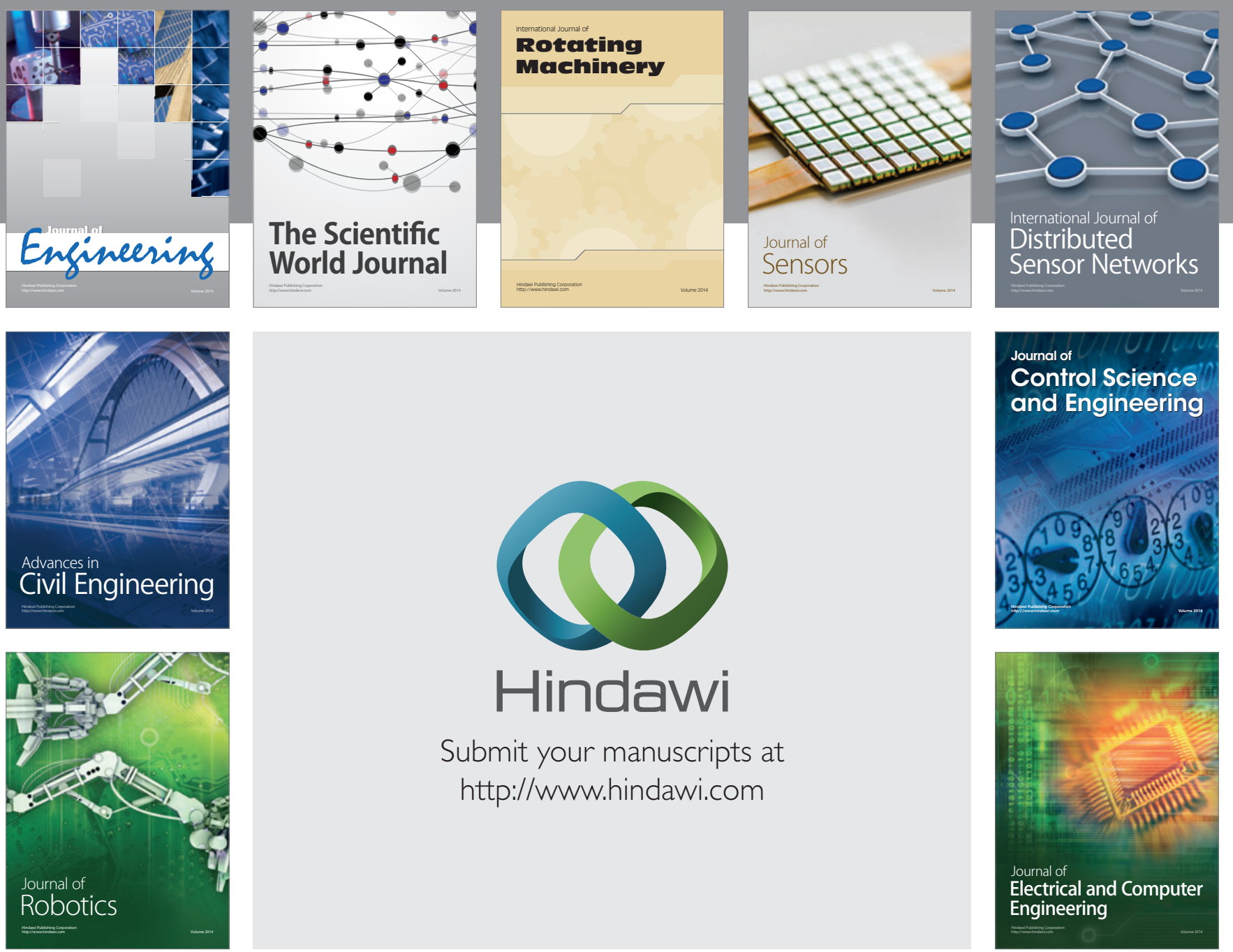

Submit your manuscripts at

http://www.hindawi.com
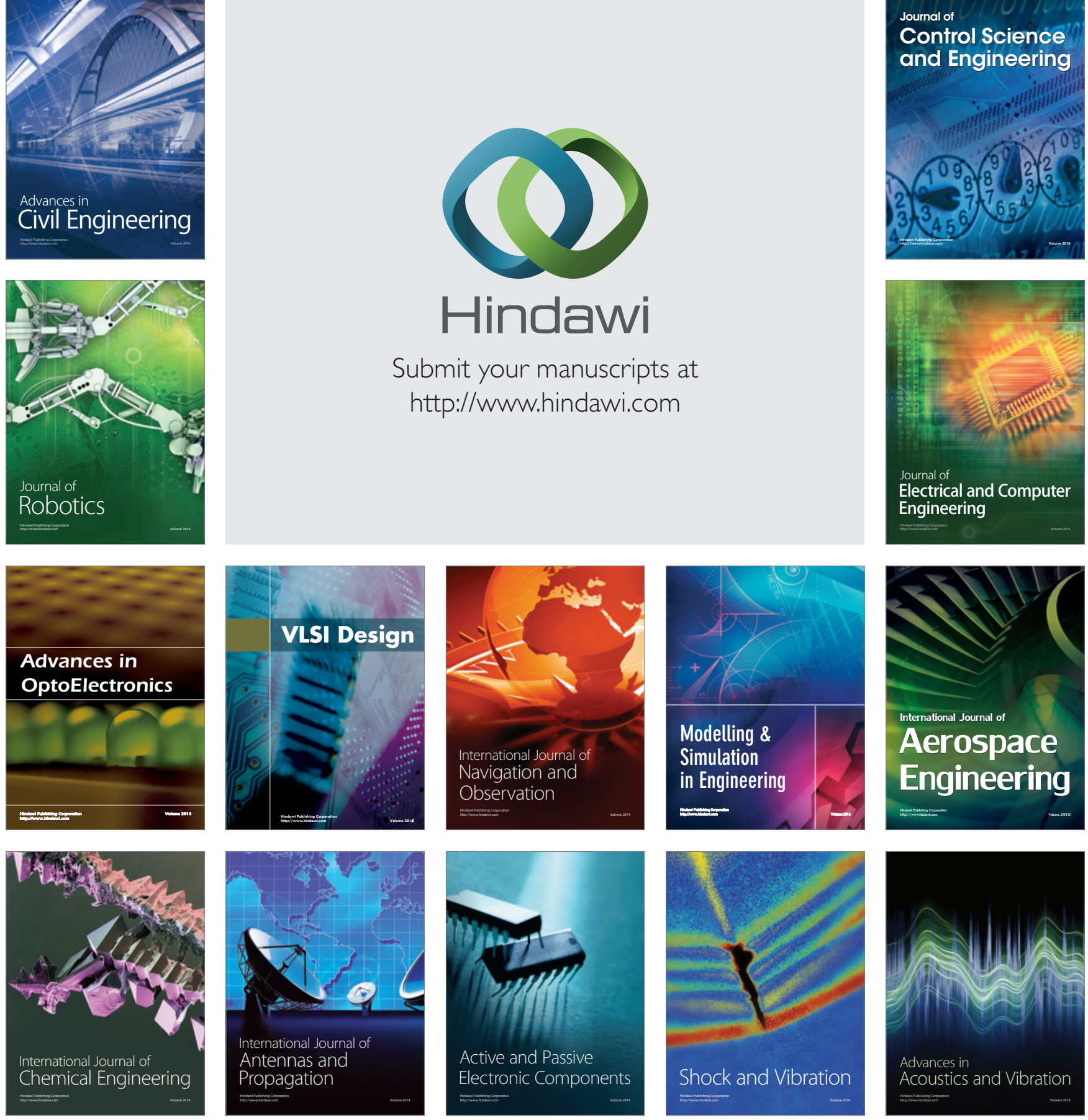